\title{
Use of extracorporeal life support for active rewarming in a hypothermic, nonarrested patient with multiple trauma
}

\author{
Daniel K. Ting MD, Douglas J.A. Brown MD
}

Cite as: CMAJ 2018 June 11;190:E718-21. doi: 10.1503/cmaj.180117

A 53-year-old man was driving in the winter when he was involved in a single-vehicle collision in the early morning hours (around 4:30). The vehicle travelled down a sixmetre embankment and struck several large trees. The man was not wearing a seatbelt and was ejected through the windshield, ending up pinned under a tree. The ambient temperature was about $-20^{\circ} \mathrm{C}$.

Loggers found the patient at 6:30 am with a decreased level of consciousness. While extrication efforts were underway, the ambulance service was notified and arrived on scene at 9 am.

The initial assessment by the paramedics documented a Glasgow Coma Scale score of 8 , a heart rate of 42 , and a blood pressure of $61 / 43$. They placed a tibial intraosseous line and intubated the patient under direct laryngoscopy. They measured an esophageal temperature of $23.5^{\circ} \mathrm{C}$. The patient was transported via air ambulance under full spinal precautions to the regional trauma and extracorporeal life support centre. Paramedics began passive rewarming techniques and also infused a litre of warmed normal saline. Prehospital notification was sent to cardiac surgery, anesthesia and intensive care.

At 11:15 am, the patient arrived at the emergency department and was assessed concurrently by the trauma team leader, cardiac surgeon, anesthesiologist and intensivist. Vital signs included a Glasgow Coma Scale score of $2 \mathrm{~T}$, a heart rate of 61 , a blood pressure of $60 / 39$ and an esophageal temperature of $24.9^{\circ} \mathrm{C}$. Primary and secondary surveys did not show any life-threatening injuries. Extended focused assessment with sonography for trauma was negative for pneumothorax, hemothorax, abdominal free fluid or pericardial effusion. Continuous cardiac monitoring showed intermittent premature ventricular complexes, but no other cardiac arrhythmias. Initial laboratory investigations showed a hemoglobin of $130 \mathrm{~g} / \mathrm{L}$ (normal range $135-169 \mathrm{~g} / \mathrm{L}$ ), a lactate of $8.5 \mathrm{mmol} / \mathrm{L}$ (normal range $0.7-2.1 \mathrm{mmol} / \mathrm{L}$ ) and a serum potassium of $3 \mathrm{mmol} / \mathrm{L}$ (normal range $3.4-4.5 \mathrm{mmol} / \mathrm{L}$ ). Results of arterial blood gas were $\mathrm{pH} 7.06$, partial pressure of carbon dioxide 53 and partial pressure of oxygen 428 on $100 \%$ oxygen. The patient's ethanol level was $50 \mathrm{mmol} / \mathrm{L}$ (he had a history of alcoholic liver cirrhosis). While in the emergency department, the patient received a second litre of warmed normal saline. The

\section{KEY POINTS}

- Extracorporeal life support can be used both for cardiopulmonary support and to rewarm patients who have had multiple trauma, either during surgical or endovascular procedures, or after major bleeding has been excluded.

- Extracorporeal life support is resource intensive and invasive; in nonarrested patients with severe hypothermia, there are no consensus criteria to guide its use, but current evidence suggests that patients who are unstable, comorbid or older are likely to benefit.

- The decision to begin extracorporeal life support in these patients must be weighed carefully against the risks of complications arising from the treatment.

- More study is required to determine optimal protocols and who may best benefit.

team decided to proceed directly to the hybrid operating room (which contains advanced imaging modalities within the surgical theatre) to facilitate further imaging, resuscitation and primary rewarming using veno-arterial extracorporeal life support.

In the hybrid operating room, an intraoperative chest radiograph, pelvis radiograph and a computed tomography scan of the patient's head were negative for acute injury. Systolic blood pressures remained below $80 \mathrm{~mm} \mathrm{Hg}$ and core temperature at $25.9^{\circ} \mathrm{C}$. The cardiac surgeon inserted a $25-\mathrm{Fr}$ venous and $19-\mathrm{Fr}$ arterial cannula into the right femoral area and started extracorporeal life support at 12:25 pm. The extracorporeal life support circuit was programmed to have flow rates of 4.5 to $5 \mathrm{~L} / \mathrm{min}$, a temperature gradient of $1-2^{\circ} \mathrm{C}$, and a fraction of inspired oxygen of $100 \%$. The team decided to avoid using heparin, as the patient had sufficient physiologic fibrinolysis from hypothermia and trauma. The patient was rewarmed to $36.5^{\circ} \mathrm{C}$ over two hours $\left(5.3^{\circ} \mathrm{C} / \mathrm{h}\right)$ and was then weaned and decannulated. Intravenous infusions of norepinephrine (0.02 to $0.12 \mu \mathrm{g} / \mathrm{kg} / \mathrm{min}$ ) and vasopressin ( 0.06 units $/ \mathrm{min}$ ) were used during extracorporeal life support. No substantial arrhythmias occurred. Four units of crystalloid and three units of packed red blood cells were transfused to support intravascular volume. 
The patient was transferred in stable condition on vasopressor support to the intensive care unit (ICU) for care and full trauma workup. He remained in the ICU for 11 days, and was discharged from hospital 33 days after admission. His trauma workup showed a C4-C5 ligamentous injury that required neurosurgical stabilization and severe frostbite to his right hand, which eventually necessitated partial amputation of all digits. The patient had a full neurologic recovery. He declined referral for treatment of his alcohol disorder.

\section{Discussion}

The choice of rewarming treatments for hypothermic patients varies from centre to centre and from physician to physician. ${ }^{1,2}$ There is not sufficient available literature to guide management of hypothermic patients, as studies are mostly retrospective in nature and limited by small patient numbers. Physiologic studies in humans are generally restricted from cooling below $35^{\circ} \mathrm{C}$ for ethical reasons, and there are no good animal models. ${ }^{3}$

Expert opinion suggests using active external and minimally invasive rewarming methods for patients who are stable, and using extracorporeal life support for patients in cardiac arrest. ${ }^{4,5}$ Nonarrested patients with severe hypothermia are at risk of cardiac arrest and therefore may benefit from transport to a centre capable of providing extracorporeal life support, rather than the closest emergency department., ${ }^{4,5}$

For nonarrested patients with severe hypothermia, it is unknown which patients may benefit from extracorporeal life support rewarming and which patients will rewarm safely with the use of minimally invasive techniques. Morita and colleagues ${ }^{6}$ conducted a historical before and after trial that included 68 nonarrested patients with core temperatures less than $28^{\circ} \mathrm{C}$. They found a $30 \%$ absolute reduction in mortality after the implementation of protocols based on extracorporeal life support for this population compared with conventional rewarming methods. Röggla and colleagues ${ }^{7}$ carried out a retrospective cohort study in an urban setting that identified 36 hypothermia patients with core temperatures less than $28^{\circ} \mathrm{C}$ who were rewarmed using conventional methods; patients had an in-hospital mortality of $42 \%$, which increased to $65 \%$ for the subgroup with systolic blood pressures less than $70 \mathrm{~mm} \mathrm{Hg}$. They showed an $8 \%$ rate of in-hospital cardiac arrest during conventional rewarming and attributed death to comorbid illness for 13 of the 15 fatalities. In contrast, younger populations with fewer comorbidities may rewarm safely with minimally invasive techniques. Wanschler and colleagues ${ }^{8}$ reported a case series in which three young nonarrested patients had core temperatures of less than or equal to $28^{\circ} \mathrm{C}$ and were rewarmed with conventional techniques without complications. Furthermore, Steele and colleagues ${ }^{9}$ prospectively studied 16 adult patients with temperatures lower than $32^{\circ} \mathrm{C}$ who were all safely rewarmed noninvasively.

In severe hypothermia, minimally invasive rewarming techniques take a median of 10 hours. $^{7}$ During this time, patients are at risk of hypothermic complications such as arrhythmia, coagulopathy and multi-organ failure. ${ }^{5}$ Extracorporeal life support rewarming is more rapid and provides cardiorespiratory support that ameliorates the poor organ perfusion associated with severe hypothermia, but it is an invasive, resource-intensive procedure with an approximate $1.2 \%$ risk of death or limb amputation. ${ }^{10}$ In 2016, the International Commission for Mountain Emergency Medicine endorsed a review that proposed five potential criteria for extracorporeal life support in nonarrested hypothermic patients ${ }^{5}$ (Box 1 ), but other experts support different criteria, including a core temperature below $28^{\circ} \mathrm{C}^{10}$ and comorbid illness that impairs tolerance for hypothermic multiorgan dysfunction. ${ }^{11}$

The clinician has limited existing evidence to guide management of patients with both hypothermia and trauma. Hypothermia in trauma is independently associated with a threefold increase in mortality, ${ }^{12,13}$ and survival may be particularly poor with mechanisms involving water submersion. ${ }^{4}$ Any hemorrhage is worsened by coagulopathy, which develops below $34^{\circ} \mathrm{C}$ and is not measured by standard assays. ${ }^{3}$ Furthermore, lactic acidosis and hypoperfusion secondary to hypovolemic shock are all poorly tolerated in trauma and contribute to patient harm. ${ }^{5}$ For these reasons, multiple trauma may be a comorbidity that prevents patients from being able to tolerate the multi-organ dysfunction associated with their hypothermia safely.

Before the development of heparin-free protocols, the use of extracorporeal life support in patients with trauma was limited. More recently, patients with trauma who have refractory hypoxemia or hypotension are being managed with extracorporeal life support while undergoing damage control through surgical or endovascular treatments. ${ }^{13}$ In such patients, the presence or absence of intracranial hemorrhage as well as the exclusion of terminal illness may be pivotal to determining the risk versus benefit of extracorporeal life support.

Patients with severe hypothermia in addition to their trauma, such as the case presented, pose unique decisionmaking challenges. Generally, such patients have a substantial traumatic mechanism, are immobile or unable to seek care for a prolonged period, have a decreased level of consciousness and are hypotensive. By the very nature of surviving long enough to become severely hypothermic, many of these patients have declared that they do not have hemorrhagic injuries that require urgent intervention, despite their hypotension and mechanism. Accurate core temperature measurement showing mild hypothermia can help rule out hypothermia as the cause of a decreased level of consciousness, but

Box 1: Selected proposed criteria for extracorporeal life support rewarming in severe hypothermia*

- Failure to improve with external active and minimally invasive rewarming methods

- Life-threatening arrhythmia

- Hypotension (systolic blood pressure $<90 \mathrm{~mm} \mathrm{Hg}$ )

- Respiratory failure

- Refractory acidosis

${ }^{*}$ As endorsed by the International Commission for Mountain Emergency Medicine. ${ }^{5}$ 
below about $29^{\circ} \mathrm{C}$, hypothermia usually causes neurologic impairment and can mask traumatic brain injury or comorbid illness. ${ }^{5}$ For trauma management, the Airway, Breathing, Circulation, Disability, Exposure $(A B C D E)$ approach should be prioritized, including temperature management and surgical or endovascular control of hemorrhage as required. Transfer to the closest appropriate trauma centre is usually indicated, regardless of core temperature. ${ }^{4}$

\section{Diagnosis}

Accidental hypothermia is defined as an involuntary drop in core temperature below $35^{\circ} \mathrm{C}$. ${ }^{4}$ Accidental hypothermia can be subdivided into primary hypothermia, caused by environmental exposure, or secondary hypothermia, caused by impaired thermoregulation. Hypothermia slows metabolism, markedly decreases oxygen consumption, and is neuroprotective, allowing for remarkable cases of survival, ${ }^{5}$ but also causes altered level of consciousness, coagulopathy and progressive multi-organ failure. Patient survival with fully intact neurologic recovery has been reported with core temperatures as low as $13.7^{\circ} \mathrm{C}$ and over six hours of cardiopulmonary resuscitation. ${ }^{5}$ Many clinicians now classify and treat hypothermia using the modified staging system: mild $\left(\mathrm{HT} \mathrm{I}, 35^{\circ} \mathrm{C}\right.$ to $\left.32^{\circ} \mathrm{C}\right)$, moderate $\left(\mathrm{HT} \mathrm{II}, 32^{\circ} \mathrm{C}\right.$ to $28^{\circ} \mathrm{C}$ ), severe (HT III, below $28^{\circ} \mathrm{C}$ ) and cardiac arrest (HT IV) (Box 2). The main advantage of this classification is that an accurate core temperature is not needed to triage transport. ${ }^{4}$ Given the multidisciplinary nature of hypothermic patient care and the relative infrequency with which providers manage hypothermia, a clinical practice guideline such as the one developed in British Columbia ${ }^{14}$ may help improve quality of care.

\section{Management}

In this case, the patient presented with severe hypothermia (core temperature of $23.5^{\circ} \mathrm{C}$ ), likely owing to a combination of primary exposure and secondary hypothermia from trauma and ethanol intoxication. Hypothermia was probably the main contributor to the profound hypotension (61/43) given the 4.5 hours since initial injury and the negative primary and secondary surveys, including the extended focused assessment with sonography for trauma (EFAST) examination; however, the severe mechanism and lack of imaging created uncertainty regarding potential traumatic injuries. The clinicians were faced with a clinical decision to do one of the following: resuscitate and rewarm in the emergency department using minimally invasive techniques or extracorporeal life support; transfer to radiology for trauma imaging before determining patient disposition; or proceed directly to the operating room for extracorporeal life support rewarming with or without trauma laparotomy. With minimally invasive rewarming, the patient would be at risk of ischemic injury from prolonged hypotension and circulatory decompensation from severe hypothermia for many hours. Proceeding directly to extracorporeal life support involved a risk of futility should the patient have already suffered a catastrophic intracranial injury. Whereas it would be ideal to image all patients with multiple trauma fully, the clinicians judged that the patient's need for ongoing resuscitation and the risk of cardiac arrest were a contraindication to imaging in the radiology department, as it is a suboptimal location for resuscitation. In this case, the operating room, where it is possible to diagnose and treat life-threatening pathology simultaneously, was selected as the optimal location for further treatment.

Box 2: Staging and treatment of accidental hypothermia ${ }^{11}$

\begin{tabular}{|llll}
\hline Stage & \multicolumn{1}{c}{ Clinical signs } & $\begin{array}{c}\text { Approximate core } \\
\text { temperature }\left({ }^{\circ} \mathrm{C}\right)\end{array}$ & \multicolumn{1}{c}{ Treatment } \\
\hline Mild (HT I) & Conscious, shivering & 32 to 35 & $\begin{array}{l}\text { - Warm environment and clothing; warm, sweet drinks; active } \\
\text { movement if possible }\end{array}$ \\
\hline Moderate (HT II) & $\begin{array}{l}\text { Impaired } \\
\text { consciousness, with or } \\
\text { without shivering }\end{array}$ & 28 to $<32$ & - Active external and minimally invasive rewarming \\
& & - Cardiac and core temperature monitoring
\end{tabular}

Note: $\mathrm{CPR}=$ cardiopulmonary resuscitation, $\mathrm{ECLS}=$ extracorporeal life support, $\mathrm{HT}$ = hypothermia.

*Refer to Box 1 for proposed ECLS criteria. 
It is impossible to know how the patient would have done without extracorporeal life support, but given his low core temperature, he was at high risk for hypothermic cardiac arrest and had a threefold increased chance of death from any traumatic injuries. ${ }^{12}$ The patient was later found to have an unstable cervical spine injury which, in the event of a malignant arrhythmia, might have been worsened by cardiopulmonary resuscitation.

\section{Conclusion}

We report the case of a nonarrested patient with severe accidental hypothermia and multiple trauma who had a full neurologic recovery after undergoing extracorporeal life support for primary rewarming. The decision to begin extracorporeal life support in patients like this must be weighed carefully against the risks of complications arising from the treatment. We suggest further research to determine the indications and contraindications for extracorporeal life support in hypothermic patients with multiple trauma.

\section{References}

1. Schober A, Sterz F, Handler C, et al. Cardiac arrest due to accidental hypothermia a 20 year review of a rare condition in an urban area. Resuscitation 2014;85:749-56.

2. van der Ploeg GJ, Goslings JC, Walpoth $\mathrm{BH}$, et al. Accidental hypothermia: rewarming treatments, complications and outcomes from one university medical centre. Resuscitation 2010;81:1550-5.

3. Danzl DF. Accidental hypothermia. In: Auerbach PS, Cushing TA, Harris NS, editors. Auerbach's Wilderness Medicine. 7th ed. Milton (ON): Elsevier; 2016:135-62.

4. Brown DJ, Brugger H, Boyd J, et al. Accidental hypothermia. N Engl J Med 2012;367:1930-8.

5. Paal P, Gordon L, Strapazzon G, et al. Accidental hypothermia - an update: the content of this review is endorsed by the International Commission for Mountain Emergency Medicine (ICAR MEDCOM). Scand J Trauma Resusc Emerg Med 2016;24:111.

6. Morita S, Inokuchi S, Yamagiwa T, et al. Efficacy of portable and percutaneous cardiopulmonary bypass rewarming versus that of conventional internal rewarming for patients with accidental deep hypothermia. Crit Care Med 2011;39:1064-8.
7. Röggla M, Frossard M, Wagner A, et al. Severe accidental hypothermia with or without hemodynamic instability: rewarming without the use of extracorporeal circulation. Wien Klin Wochenschr 2002;114:315-20.

8. Wanscher M, Agersnap L, Ravn J, et al. Outcome of accidental hypothermia with or without circulatory arrest: experience from the Danish Praesto Fjord boating accident. Resuscitation 2012;83:1078-84.

9. Steele MT, Nelson MJ, Sessler DI, et al. Forced air speeds rewarming in accidental hypothermia. Ann Emerg Med 1996;27:479-84.

10. Dunne B, Christou E, Duff O, et al. Extracorporeal-assisted rewarming in the management of accidental deep hypothermic cardiac arrest: a systematic review of the literature. Heart Lung Circ 2014;23:1029-35.

11. Brown DJ. Hypothermia. In: Tintinalli JE, Stapczynski JS, Ma OJ, et al., editors. Tintinalli's Emergency Medicine: A comprehensive study guide. 8th ed. New York: McGraw-Hill; 2015:1357-65.

12. Ireland S, Endacott R, Cameron P, et al. The incidence and significance of accidental hypothermia in major trauma - a prospective observational study. Resuscitation 2011;82:300-6.

13. Arlt M, Philipp A, Voelkel S, et al. Extracorporeal membrane oxygenation in severe trauma patients with bleeding shock. Resuscitation 2010;81:804-9.

14. Brown DJ. Accidental hypothermia clinical practice guideline for British Columbia. British Columbia Accidental Hypothermia Working Group; 2016.

\section{Competing interests: None declared.}

This article has been peer reviewed.

The authors have obtained patient consent.

Affiliations: Department of Emergency Medicine (Ting), University of British Columbia, Kelowna, BC; Department of Emergency Medicine (Brown), University of British Columbia, Vancouver BC

Contributors: Both authors contributed to the conception and design of the work, drafted the manuscript, revised it critically for important intellectual content, gave final approval of the version to be published and agreed to be accountable for all aspects of the work.

Acknowledgements: We are indebted to the interprofessional health care team at Kelowna General Hospital as well as the British Columbia Ambulance Service for their roles in patient care. In particular, we thank Drs. Nick Kuzak, Vance Beck and Paul Cleve.

Correspondence to: Daniel Ting, daniel.ting@alumni.ubc.ca 